\title{
Comparison of extubation success using noninvasive positive pressure ventilation (NIPPV) versus noninvasive neurally adjusted ventilatory assist (NI-NAVA)
}

\author{
Kartikeya Makker ${ }^{1} \cdot$ Josef Cortez $^{1} \cdot$ Kanishk Jha $^{1} \cdot$ Sanket Shah $^{1} \cdot$ Padma Nandula $^{1} \cdot$ David Lowrie $^{1}$. \\ Carmen Smotherman $^{2} \cdot$ Shiva Gautam $^{2} \cdot$ Mark L. Hudak $^{1}{ }^{1}$
}

Received: 9 September 2019 / Revised: 18 November 2019 / Accepted: 18 December 2019 / Published online: 7 January 2020

(c) The Author(s), under exclusive licence to Springer Nature America, Inc. 2020

\begin{abstract} until 12 days post extubation. successful.

\section{Introduction}

Support with mechanical ventilation is essential for the survival of many preterm infants. However, prolonged mechanical ventilation is associated with higher neonatal mortality and increases the risk for bronchopulmonary dysplasia (BPD) [1, 2]. Failure of elective extubation has been independently associated with prolonged need for oxygen and ventilator support, longer hospitalization, and increased mortality. Prolonged endotracheal intubation is a major risk factor for airway injury possibly leading to
\end{abstract}

Objective Compare rates of initial extubation success in preterm infants extubated to NIPPV or NI-NAVA.

Study design In this pilot study, we randomized 30 mechanically ventilated preterm infants at the time of initial elective extubation to NI-NAVA or NIPPV in a 1:1 assignment. Primary study outcome was initial extubation success.

Results Rates of continuous extubation for $120 \mathrm{~h}$ were $92 \%$ in the NI-NAVA group and $69 \%$ in the NIPPV group (12/13 vs. 9/13, respectively, $p=0.14$ ). Infants extubated to NI-NAVA remained extubated longer (median 18 vs. 4 days, $p=0.02$ ) and experienced lower peak inspiratory pressures (PIP) than infants managed with NIPPV throughout the first 3 days after extubation. Survival analysis through 14 days post extubation showed a sustained difference in the primary study outcome

Conclusions Our study is the first to suggest that a strategy of extubating preterm infants to NI-NAVA may be more

Supplementary information The online version of this article (https:// doi.org/10.1038/s41372-019-0578-4) contains supplementary material, which is available to authorized users.

Kartikeya Makker

kmakker@gmail.com

1 Division of Neonatology, Department of Pediatrics, University of Florida College of Medicine-Jacksonville, Jacksonville, FL, USA

2 Center for Health Equity and Research (CHEQR), University of Florida College of Medicine_Jacksonville, Jacksonville, FL, USA tracheomalacia [3]. Each additional week of intubation and mechanical ventilation has been associated with additional risk of neurodevelopmental impairment [1, 4]. Hence, a reduction in the duration of endotracheal mechanical ventilation through a strategy that facilitates earlier, safe and successful extubation might be expected to improve the overall outcome of preterm infants.

Conventional ventilation strategies are limited by imperfect synchrony between infant respirations and ventilator breaths. This asynchrony can result in an infant "fighting the ventilator" and higher requirements for inspired oxygen and ventilatory support. Preterm infants who are electively extubated to conventional modalities (e.g., nasal continuous positive pressure (nCPAP) or NIPPV) have a high likelihood of requiring reintubation [5]. Of the two, NIPPV has been shown to be superior to nCPAP in reducing the incidence of extubation failure (typical RR 0.70 , 95\% CI 0.60-0.80) and need for reintubation $48 \mathrm{~h}$ to 7 days after extubation (typical RR $0.76,95 \%$ CI 0.65-0.88). The authors concluded that synchronization may be important in delivering effective noninvasive ventilation [5]. Synchronization of an infant's respiratory effort with ventilator inflation has been associated with improved oxygenation and carbon dioxide elimination [6]. It is 
reasonable to postulate that improved synchronization of inspiratory efforts with positive pressure inflations will allow use of lower inspired oxygen concentration and PIP, reduce ongoing lung injury caused by barovolutrauma or hyperoxia, and thereby increase the duration of sustained extubation.

NAVA is a newer ventilation technology that uses the patient's respiratory drive (as quantitated by the electrical activity of the diaphragm (Edi)) to control the timing, duration, and magnitude of the PIP of each breath. NAVA has been reported to increase patient-ventilator respiratory synchrony $[7,8]$ and unload the respiratory muscles $[7,9]$. NI-NAVA has also been shown to be effective without PEEP and despite high excessive leaks $(75 \%)$ of tidal volume [10]. Optimizing NAVA management protocols holds promise to facilitate and maintain successful elective extubation and thereby reduce the total duration of ventilator support. Because extubation failure is associated with significant respiratory setback, the ability to maintain extubation in a preterm infant may have significant positive impact on an infant's overall health. However, no trials have yet compared the rates of successful initial extubation between infants managed with NI-NAVA to infants managed with nCPAP or NIPPV. In fact, only one randomized controlled trial has compared NAVA with conventional ventilatory modalities [11]. In this trial we aimed to compare the success rate of initial elective extubation in preterm infants managed after extubation on NI-NAVA and NIPPV.

\section{Materials and methods}

This prospective randomized controlled clinical study was conducted at our regional perinatal center neonatal intensive care unit at University of Florida Health, Jacksonville from October 2017 to November 2018. The Institutional Review Board of the University of Florida College of Medicine (UFCOM) Jacksonville approved this study (clinicaltrials. gov registration number NCT03242057). A Data Safety Monitoring Committee was appointed that reviewed study progress three times and adverse events and protocol deviations when required. The Data Safety Monitoring Committee reports were provided to the IRB.

\section{Inclusion criteria}

Infants born between 24 and 32 weeks' gestational age and with birth weight $\leq 1500 \mathrm{~g}$ who were intubated by $24 \mathrm{~h}$ of age were eligible for enrollment after 12 consecutive hours of mechanical ventilation. Parents of eligible infants who met study criteria for elective extubation (supplement) before 14 days of age were approached for consent.

\section{Exclusion criteria}

We excluded infants based on the following criteria: (1) a failed initial elective extubation prior to study enrollment; (2) major congenital anomalies or known/suspected chromosomal anomalies; (3) treatment with paralytics in previous $24 \mathrm{~h}$; (4) participation in another randomized interventional trial; (5) known or suspected phrenic nerve palsy or lesion; (6) known or suspected diaphragmatic lesion; and (6) any contraindication for gastric tube placement.

\section{Enrollment and randomization}

After parental consent, enrolled infants were randomized to treatment with either NI-NAVA or NIPPV strategies after extubation. We used sequentially numbered white opaque sealed envelopes with random block assignment to mask the ventilatory assignment. Blinding to intervention after randomization was not feasible.

\section{Ventilator management prior to extubation}

The initial mode of invasive ventilation (conventional mechanical ventilation, invasive NAVA, high-frequency oscillatory, or jet ventilation) was chosen by the attending physician.

Edi was measured by an array of nine miniaturized electrodes mounted on a conventional feeding tube (Maquet Critical Care AB, Solna, Sweden; Neurovent Research Inc, Toronto, Canada) whose tip was positioned in the lower esophagus at the level of the diaphragm. A NAVA catheter was placed $\sim 1 \mathrm{~h}$. prior to extubation for the infants randomized to NI-NAVA to allow time to achieve correct tube placement and to calibrate Edi signals.

\section{Study intervention}

Our study protocol defined criteria for initial elective extubation and for reintubation (supplement).

\section{Ventilator management post extubation}

NI-NAVA and NIPPV settings after initial elective extubation were managed per study protocol and subsequently adjusted as the clinical situation demanded (supplement). Ventilator management after an infant had been extubated for $120 \mathrm{~h}$ or reintubated before $120 \mathrm{~h}$ after extubation was decided by the attending physician.

\section{Criteria for reintubation}

Criteria for reintubation were predefined for our study (supplement). 


\section{Intervention crossover}

We allowed crossover between the two ventilator modalities if the infant met most but not all reintubation criteria and the provider determined that changing the ventilatory modality might forestall reintubation.

\section{Blood gas analysis}

The protocol mandated obtaining a blood gas (arterial if possible) by $4 \mathrm{~h}$ after elective extubation. Additional blood gas analysis was performed per the discretion of the clinical team.

\section{Primary study outcome}

The primary study outcome was the success of the initial elective extubation (defined as continuous extubation for $120 \mathrm{~h}$ ).

\section{Secondary outcomes}

Secondary outcomes included extubation success at 72 and $168 \mathrm{~h}$, the number of ventilator days (invasive and noninvasive), BPD, patent ductus arteriosus (PDA), retinopathy of prematurity (ROP), culture-positive sepsis, ventilatorassociated pneumonia (VAP), necrotizing enterocolitis (NEC), spontaneous intestinal perforation (SIP), abdominal distension $>2 \mathrm{~cm}$ from baseline and with signs necessitating cessation of feeds during the first $48 \mathrm{~h}$ after extubation, severe intraventricular hemorrhage, pulmonary air leak, length of NICU hospitalization, and mortality.

Gestational age was determined by the best obstetrical estimate using last menstrual period and/or an appropriately timed dating ultrasound. NEC was defined as Stage II or greater using modified Bell's criteria [12] Late-onset sepsis was defined as the presence of clinical signs of sepsis and a positive blood culture after $72 \mathrm{~h}$ of age. We defined positive pressure days as the total number of days an infant received any positive pressure (conventional ventilation, high frequency ventilation, invasive NAVA, NIPPV, or NI-NAVA, nCPAP, or HFNC $>2 \mathrm{LPM}$ ). The PIP recorded was averaged for the eight values per $24 \mathrm{~h}$. period documented in EMR. In addition, the trends of PIP for the $24 \mathrm{~h}$. period were visually screened on the NAVA ventilator to confirm that the recorded values were representative. BPD was defined as an oxygen requirement at 36 weeks' postmenstrual age. Moderate and severe BPD were defined per published criteria [13, 14]. A pediatric radiologist evaluated head ultrasounds to determine the presence and grade of intraventricular hemorrhage. Severe intraventricular hemorrhage on head ultrasound was defined as grade III or grade IV per the Papile classification [15]. We used the international classification to stage ROP [16]. VAP was defined as a deterioration in respiratory status in the setting of new radiographic findings consistent with pneumonia, a culture-positive tracheal aspirate, and initiation of antibiotic treatment by the clinical team.

\section{Statistical analysis}

We enrolled and randomized 30 infants, of which 26 received the specified intervention. Two infants randomized to each intervention arm deteriorated shortly after randomization and did not meet elective extubation criteria by 14 days of age (Fig. 1). Because we did not have historical data on extubation failure rates for infants managed in our unit with NI-NAVA or NIPPV and because this was a pilot (feasibility) study, we did not perform a power analysis. By recruiting 15 infants in each arm, we hoped to identify a sufficient difference that would justify a larger pivotal trial that could then be powered to demonstrate a clinically significant improvement in the primary efficacy outcome measure. We anticipated that it would not be possible to consent the parents of all eligible infants. We received IRB approval to collect data on these eligible but nonenrolled infants during the study period. Because the study only defined extubation and reintubation criteria and all infants (enrolled or not) were managed according to the study/ institutional protocol we felt that outcome assessment of these infants would provide additional relevant information. Statistical support was provided by the Center for Health Equity and Quality Research at UFCOM. All analyses were performed on an intent-to-treat approach. Continuous variables were summarized using means, medians, and standard deviations. Categorical variables were summarized using counts, proportions, and percentages. The primary outcome was compared between the two groups using chi-square test or Fisher's exact test. Secondary outcomes were compared using Wilcoxon rank sum test (continuous variables) or chisquare/Fisher's exact test (categorical variables). The level of significance was set at 0.05 . All analyses were performed using SAS ${ }^{\bullet}$ for Windows Version 9 (SAS ${ }^{\bullet}$ Version 9.4 for Windows, Cary, NC: SAS Institute Inc.; 2008).

\section{Results}

Figure 1 shows the Consort flow diagram for this study. During the study period, 59 infants were eligible for enrollment into this study. For 27 infants, we missed the opportunity to approach the parents for consent. In two cases, parents declined to participate. We did obtain informed consent for 30 infants. Fifteen infants were randomized to treatment with either NI-NAVA or NIPPV and 
Fig. 1 CONSORT

(Consolidated Standards of Reporting Trials) 2010 flow diagram.

\section{Study CONSORT Diagram}

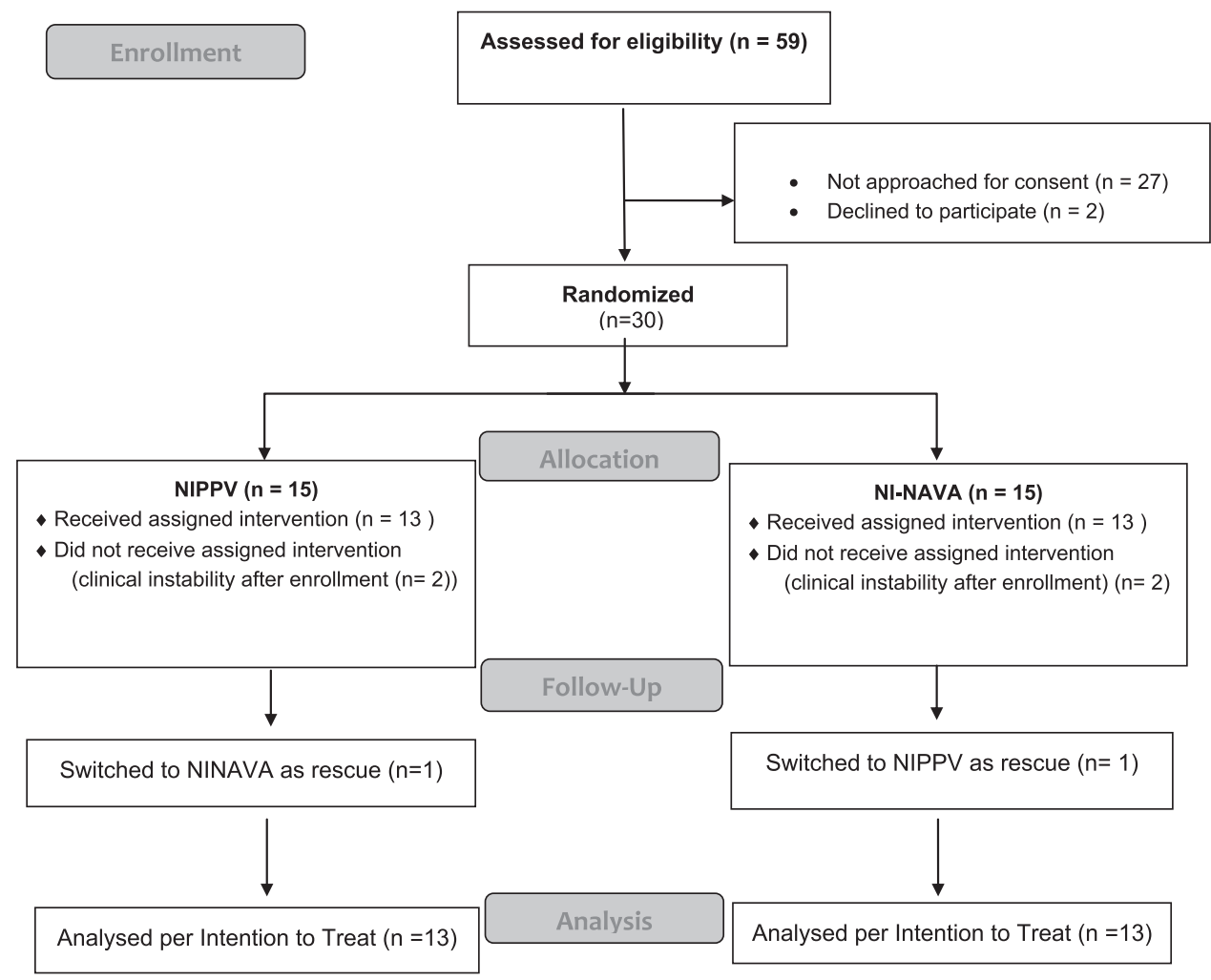

13 received the assigned intervention in each group. The other two randomized infants in each group deteriorated clinically in a short time and did not subsequently meet extubation criteria by day of life 14 (study enrollment deadline). One infant in each group of 13 crossed over to the other ventilatory mode after the initial assignment and study extubation per the decision of the attending physician.

The two groups had similar characteristics at enrollment (Table 1). All infants received caffeine prior to study extubation followed by maintenance dosing. Use of any and completion of a full course of antenatal steroids occurred with greater frequency in the NIPPV group but did not achieve statistical significance.

Table 2 summarizes the information related to the primary study outcome. Infants were extubated at a median age of 3 days of age in both groups. Twelve of 13 (92\%) infants in the NI-NAVA group compared with 9 of 13 $(69 \%)$ infants in the NIPPV group remained extubated at 72 and $120 \mathrm{~h}$ after elective extubation $(p=0.14)$. By $168 \mathrm{~h}$ after extubation, $11(85 \%)$ infants in the NI-NAVA group compared with $9(69 \%)$ infants in the NIPPV group remained extubated $(p=0.35)$. Over the course of the full hospitalization, five infants assigned to NI-NAVA and six infants assigned to NIPPV required reintubation at a median of 18 and 4 days after initial elective extubation $(p=0.02)$.
Table 3 presents details related to the ventilatory course. Infants extubated to NI-NAVA were managed with significantly less PIP during the first 3 days after extubation. In the NI-NAVA group, the median average Edi min values over the $120 \mathrm{~h}$ after extubation ranged from 1.6 to 2.1, the median average Edi peak values ranged from 6.8 to 8.5 , and the median average NAVA level ranged from 1.1 to 1.6. The median total positive pressure days (intubated and noninvasive) was 14 days in the NI-NAVA group compared with 30 days in the NIPPV group $(p=0.77)$. The median fractional oxygen concentration for all 5 days were similar in two groups with average concentrations below $40 \%$ in both groups (NI-NAVA median range $21-30 \%$ and NIPPV median range $27-32 \%$ ).

Secondary outcomes related to safety were similar in the two groups (Table 4). One infant in each group developed abdominal distension that resulted in cessation of feedings within $48 \mathrm{~h}$ of extubation. There were no SIPs and one episode of NEC stage 2 or greater in each group. Moderate to severe BPD, use of diuretics and of postnatal steroids for treatment of BPD were similar. Anthropometric measurements at 36 weeks' postmenstrual age were also similar (Table 4).

A Kaplan-Meier analysis showed a higher probability of extubation success in NI-NAVA through 12 days post 
Table 1 Infant characteristics.

\begin{tabular}{|c|c|c|c|}
\hline & NAVA $(n=13)$ & $\operatorname{NIPPV}(n=13)$ & $P$ value \\
\hline Birth weight $(\mathrm{g})^{\mathrm{a}}$ & $1000(840,1120)$ & $990(690,1370)$ & $0.90 * *$ \\
\hline Gestational age (weeks) ${ }^{\mathrm{a}}$ & $27(25,28)$ & $27(26,30)$ & $0.89 * *$ \\
\hline Male gender & $6(46)$ & $5(38)$ & $0.69 *$ \\
\hline African-American & $9(69)$ & $9(69)$ & $1.00 *$ \\
\hline Inborn & $11(85)$ & $12(92)$ & $0.54 *$ \\
\hline C section & $10(77)$ & $8(62)$ & $0.40 *$ \\
\hline APGAR score $1 \mathrm{~min}^{\mathrm{a}}$ & $5(2,6)$ & $4(2,6)$ & $0.88 * *$ \\
\hline APGAR score $5 \mathrm{~min}^{\mathrm{a}}$ & $7(6,8)$ & $7(6,8)$ & $0.69 * *$ \\
\hline APGAR 10 min if available & $6(6,8)(n=5)$ & $6(6,7)(n=6)$ & $0.78 * *$ \\
\hline Number of surfactant doses ${ }^{a}$ & $1(1,2)$ & $1(1,2)$ & $1.00 * *$ \\
\hline Use of any antenatal steroid & $8(62)$ & $11(85)$ & $0.38 * * *$ \\
\hline Use of completed course of antenatal steroids & $4(31)$ & $8(62)$ & $0.23 * * *$ \\
\hline Number of doses of antenatal steroid used ${ }^{\mathrm{a}}$ & $1(0,2)$ & $2(1,2)$ & $0.18 * *$ \\
\hline Caffeine use & $13(100)$ & $13(100)$ & $1.00 *$ \\
\hline
\end{tabular}

Data are presented as counts (percentages) unless specified

*Pearson's Chi-square; **Wilcoxon rank sum test, ${ }^{\#} p<0.05$, ***Fisher's exact

${ }^{a}$ Median (1st quartile, 3rd quartile)

Table 2 Respiratory outcomes.

\begin{tabular}{|c|c|c|c|}
\hline & NAVA $(n=13)$ & $\operatorname{NIPPV}(n=13)$ & $P$ value \\
\hline DOL first elective study extubation ${ }^{\mathrm{a}}$ & $3(3,5)$ & $3(2,5)$ & $0.48 * *$ \\
\hline Extubation success at $120 \mathrm{~h}$ post extubation & $12(92)$ & $9(69)$ & $0.14^{*}$ \\
\hline DOL first reintubation after elective study extubation ${ }^{\mathrm{a}}$ & $18(14,23)$ & $4(3,6)$ & $0.02 * * \#$ \\
\hline
\end{tabular}

Data are presented as counts (percentages) unless specified

$D O L$ day of life

*Pearson's Chi-square; **Wilcoxon rank sum test, ${ }^{*} p<0.05$

${ }^{a}$ Median (1st quartile, 3rd quartile)

extubation. This is based on small numbers for this study (Fig. 2).

Of the 27 eligible infants for whom consent was not attempted, 4 infants were extubated per provider discretion to nCPAP. We abstracted outcome data for the remaining 23 infants (16 extubated to NIPPV and 7 to NI-NAVA). We observed continuous extubation through $120 \mathrm{~h}$ in $86 \%(6 / 7)$ of the infants managed initially on NI-NAVA compared with $75 \%(12 / 16)$ of infants managed with NIPPV ( $p=$ $0.4)$.

\section{Discussion}

Mechanically ventilated preterm infants are at risk for multiple morbidities and the absolute risk increases with each additional week of ventilation [4, 17]. A reduction in the use and/or duration of invasive mechanical ventilation may potentially improve individual and composite outcomes of preterm infants. Ideally, this goal may be achieved by preferential initiation of noninvasive respiratory support and, for infants intubated at birth, by earlier successful elective extubation. Clinicians will gravitate to ventilatory strategies that are demonstrated to forestall invasive ventilation, facilitate earlier elective intubation, and/or reduce the total days of invasive ventilatory support [18]. A recent Cochrane review compared the effectiveness of NIPPV and nCPAP after extubation [5]. The meta-analyses demonstrated a statistically and clinically significant reduction in the risk of meeting extubation failure criteria (typical RR 0.70 , NNTB 8, 95\% CI 6-13) and subsequent reintubation (typical RR 0.76 , NNTB 10, 95\% CI 7-20) in favor of NIPPV [5].

Compared with other modes of triggered ventilation, NAVA may improve breath-to-breath synchrony of the initiation and termination of each ventilator cycle with an infant's spontaneous respiratory activity [19, 20]. More effective synchronization using NAVA may allow adequate gas exchange to occur at lower peak airway pressures and hence NAVA might be expected to reduce cumulative lung barovolutrauma. Preliminary studies of NAVA have been promising but randomized controlled trials (RCT) to 
Table 3 Ventilator support outcomes.

\begin{tabular}{|c|c|c|c|}
\hline & NI-NAVA $(n=13)$ & $\operatorname{NIPPV}(n=13)$ & $P$ value \\
\hline PIP day 1 of extubation ${ }^{\mathrm{a}}$ & $15(13,20)$ & $21(20,22)$ & $0.009 * * \#$ \\
\hline PIP day 2 of extubation ${ }^{a}$ & $16(15,20)$ & $20(20,22)$ & $0.004 * * \#$ \\
\hline PIP day 3 of extubation ${ }^{a}$ & $16(16,18)(n=11)$ & $20(20,22)(n=10)$ & $0.004 * * \#$ \\
\hline PIP day 4 of extubation ${ }^{a}$ & $17(16,20)(n=8)$ & $20(18,21)(n=7)$ & $0.08 * *$ \\
\hline PIP day 5 of extubation ${ }^{a}$ & $15(14,17)(n=6)$ & $22(18,22)(n=5)$ & $0.09 * *$ \\
\hline Invasive CMV and HFV days post study extubation ${ }^{\mathrm{a}}$ & $0(0,12)$ & $0(0,8)$ & $0.67 * *$ \\
\hline $\begin{array}{l}\text { Noninvasive conventional positive pressure days post study extubation }{ }^{\mathrm{a}} \text { (CPAP, HFNC } \\
>2 \mathrm{lpm} \text {, NIPPV) }\end{array}$ & $11(3,28)$ & $21(6,32)$ & $0.32 * *$ \\
\hline Invasive NAVA days post study extubation ${ }^{\mathrm{a}}$ & $0(0,0)$ & $0(0,2)$ & $0.49 * *$ \\
\hline Noninvasive NAVA days after study extubation ${ }^{\mathrm{a}}$ & $4(3,10)$ & $0(0,2)$ & $0.001 * *$ \\
\hline Invasive positive pressure days post study extubation $(\mathrm{NAVA}+\mathrm{CMV}+\mathrm{HF})^{\mathrm{a}}$ & $0(0,17)$ & $0(0,13)$ & $0.81 * *$ \\
\hline $\begin{array}{l}\text { Noninvasive positive pressure days post study extubation (NI-NAVA }+ \text { CPAP }+ \text { NIPPV } \\
+\mathrm{HFNC}>2 \mathrm{lpm})^{\mathrm{a}}\end{array}$ & $14(6,41)$ & $29(6,32)$ & $0.72 * *$ \\
\hline Total positive pressure days post study extubation ${ }^{\mathrm{a}}$ & $14(6,63)$ & $30(8,49)$ & $0.77 * *$ \\
\hline
\end{tabular}

Data are presented as counts (percentages) unless specified

$C M V$ conventional mechanical ventilation, $H F V$ high frequency ventilation, $H F N C$ high flow nasal cannula

*Pearson's Chi-square; **Wilcoxon rank sum test, ${ }^{*} p<0.05$, ***Fisher's exact

${ }^{\mathrm{a}}$ Median (1st quartile, 3rd quartile)

Table 4 Clinical outcomes.

\begin{tabular}{|c|c|c|c|}
\hline & NI-NAVA $(n=13)$ & $\operatorname{NIPPV}(n=13)$ & $P$ value \\
\hline Sepsis & $0(0)$ & $1(8)$ & $0.25 *$ \\
\hline Pneumonia & $2(15)$ & $1(8)$ & $0.25 *$ \\
\hline IVH grade $3 / 4$ & $1(8)$ & $1(8)$ & $1.0 * * *$ \\
\hline Air leaks & $0(0)$ & $1(8)$ & $0.31 *$ \\
\hline BPD moderate/severe & $4(31)$ & $6(46)$ & $0.42 *$ \\
\hline NEC ( $\geq$ stage 2 ) & $1(8)$ & $1(8)$ & $1.00 *$ \\
\hline PDA moderate/large & $6(46)$ & $5(42)$ & $0.82 *$ \\
\hline PDA (medically treated) & $2(15)$ & $2(15)$ & $0.96^{*}$ \\
\hline ROP $>$ stage 2 & $3(23)$ & $2(15)$ & $0.62 *$ \\
\hline Days of caffeine use ${ }^{a}$ & $42(29,49)$ & $41(24,50)$ & $0.57 * *$ \\
\hline Use of chronic diuretics post study extubation & $5(38)$ & $5(38)$ & $0.58 *$ \\
\hline Days on chronic diuretics post study extubation ${ }^{a}$ & $58(18,60)$ & $18(15,39)$ & $0.30^{* *}$ \\
\hline Steroid use post study extubation & $4(31)$ & $2(15)$ & $0.35 *$ \\
\hline Days of steroid use post study extubation ${ }^{\mathrm{a}}$ & $10(10,15)$ & $8(8,10)$ & $0.17 * *$ \\
\hline Length of stay ${ }^{a}$ & $78(58,87)$ & $67(56,99)$ & $0.71 * *$ \\
\hline Weight $(\mathrm{g})$ at 36 weeks' PMA $^{\mathrm{a}}$ & $2134(1980,2357)$ & $1978(1823,2140)$ & $0.24 * *$ \\
\hline Length $(\mathrm{cm})$ at 36 weeks' $\mathrm{PMA}^{\mathrm{a}}$ & $44(42,46)$ & $43(41,44)$ & $0.27 * *$ \\
\hline Head circumference $(\mathrm{cm})$ at 36 weeks' $\mathrm{PMA}^{\mathrm{a}}$ & $31(30,31.5)$ & $30.5(29,31)$ & $0.53 * *$ \\
\hline
\end{tabular}

Data are presented as counts (percentages) unless specified

$P M A$ postmenstrual age

*Pearson's Chi-square; **Wilcoxon rank sum test, ${ }^{\#} p<0.05$, ***Fisher's exact

${ }^{a}$ Median (1st quartile, 3rd quartile)

confirm this efficacy are still limited. A recent Cochrane review found only one RCT that compared NAVA to patient-triggered time-cycled pressure-limited ventilation and found no significant difference in duration of mechanical ventilation or, rates of BPD, pneumothorax, or IVH [11]. This study reported lower peak PIP in the NAVA arm. This trial included moderately preterm infants (28-36 completed weeks' gestational age) and did not report any 


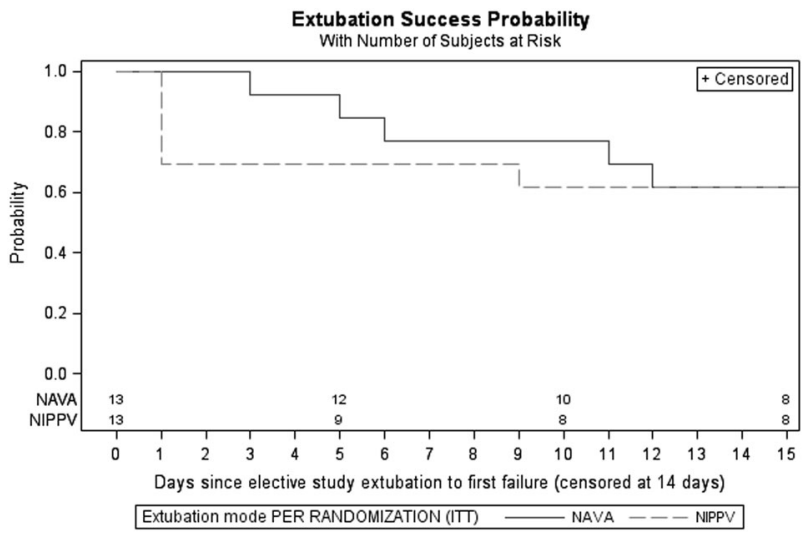

Fig. 2 Kaplan-Meier survival curve/failure time data analysis depicting the probability of successful extubation through 14 days from initial elective extubation.

data specifically related to the success of elective extubation [21]. To our knowledge, no trial has compared the rates of successful elective extubation in infants managed with NINAVA vs infants managed with conventional ventilator modalities after extubation. Our study is the first reported RCT that has compared NI-NAVA to NIPPV with respect to achieving a successful initial elective extubation in preterm infants as young as 24 weeks' gestational age.

Preterm infants randomized to NAVA in this small pilot study had a $92 \%$ rate of successful extubation at $120 \mathrm{~h}$ compared with a rate of $69 \%$ among infants randomized to NIPPV (12/13 versus $9 / 13$, respectively, $p=0.14)$. These rates equalized by 12 days after elective extubation. Our study adopted "successful extubation" as a primary outcome defined as continuous extubation for $120 \mathrm{~h}$ after the initial elective extubation. This definition is consistent with the one adopted in a secondary analysis of the National Institute of Child Health and Human Development Neonatal Research Network's Surfactant, Positive Pressure, and Oxygenation Randomized Trial [1]. However, no consensus exists on a common definition of what duration of sustained extubation would best define extubation success, with reported durations ranging from $72 \mathrm{~h}$ to 7 days [22]. A longer window of observation would identify a greater number of reintubated infants but in many cases later reintubation is done for reasons unrelated to the initial respiratory condition (e.g., late-onset sepsis and NEC). In fact, a recent systematic review of studies undertaken to define the ideal duration of extubation success in preterm infants less than $1000 \mathrm{~g}$ at birth did document that reintubation rates steadily increased with prolongation of the window of observation without any plateau $(p=0.001)$ [23].

Prevention of reintubation of preterm infants holds significant promise in improving overall outcomes of this fragile population. Cohort studies have reported that almost half of infants with birth weights less than $1500 \mathrm{~g}$ and almost two-thirds of infants with birth weights less than $1000 \mathrm{~g}$ require at least one reintubation for resumption of invasive positive pressure ventilation during the NICU stay [24-26]. The time interval between an extubation and resumption of mechanical ventilation has been reported to range from hours to days [24].

The independent impact of reintubation on survival and respiratory outcomes is not well defined. However recent retrospective sub analyses from two large RCTs showed that reintubation within 5 and 7 days after extubation independently increased respiratory morbidities and mortality [27]. In another exploratory analysis of an ongoing multicenter trial, the authors reported that after adjusting for confounders, reintubation within observation windows ranging between $24 \mathrm{~h}$ and 3 weeks after extubation was associated with an increased odds ratio of death and/or BPD (but not BPD alone), independent of the total duration of mechanical ventilation, with the greatest risk occurring among infants reintubated within the first $48 \mathrm{~h}$ after extubation [1, 24].

In addition, another retrospective cohort study found that exposure to a greater number of courses of mechanical ventilation was associated with increasing risk of BPD and the use of supplemental oxygen at discharge. Compared with a single course of ventilation, the adjusted odds ratios for BPD ranged from 1.88 (95\% CI, 1.54-2.31) among infants with two ventilation courses to 3.81 (95\% CI, 2.885.04) among those with four or more courses [28]. After adjustment for the cumulative duration of mechanical ventilation, the odds ratio for BPD was only increased among infants exposed to four or more ventilation courses (adjusted odds ratio, 1.44; 95\% CI, 1.04-2.01). These authors concluded that clinicians should attempt to extubate extremely low birth infants managed on low ventilator settings, even when success is not guaranteed [28]. Similarly, an earlier study of 262 preterm infants with gestational age $\leq 28$ weeks intubated on day of life 1 noted that infants extubated after 7 days of age were more likely to develop BPD or to meet the combined outcome of BPD and/or death. Infants who were extubated early but required later reintubation had a lower incidence of BPD/death compared with infants who were extubated later [29]. In our study, the time to first reintubation after the initial elective extubation was a median of 18 days in the NI-NAVA group and a median of 4 days in the NIPPV group. A larger study to determine if these results are reproducible and generalizable could also amplify discussion of a possible association between timeliness of initial extubation and risk of BPD or death and/or BPD.

Mechanical ventilation is an established risk factor for BPD. Alveolar overdistention, intra- and extra-pulmonary air leaks and injury to small airway epithelium are manifestations of barovolutrauma caused by invasive mechanical 
ventilation [30]. Damage to the vocal cords and subglottic area as well as abnormal tracheal development are potential adverse effects of an indwelling endotracheal tube. Ventilation strategies in preterm infants that aim to protect the lung and small airways from injury have in common the goals to minimize ventilator pressures and achieve physiologically appropriate tidal volumes. Timely removal of the endotracheal tube that results in sustained extubation will reduce the risk of injury to the large airways and vocal cords and surrounding structures. In this light, it is notable that study infants extubated to NI-NAVA were managed with significantly lower PIPs than infants managed with NIPPV. This finding is consistent with previous studies that have shown that preterm infants on invasive NAVA were managed on lower PIP compared with conventional ventilation $[19,31]$. One of these studies that used a crossover design showed that NAVA-ventilated infants also were managed with lower $\mathrm{FiO}_{2}$ and IMV rate, achieved a lower $\mathrm{PCO}_{2}$, and demonstrated greater compliance compared with pressurecontrolled ventilation [31]. A second randomized crossover study reported that PIP and work of breathing, but not the fraction of inspired oxygen or mean airway pressure, were lower for infants on NAVA compared with infants managed with conventional ventilation [32]. The only other randomized trial that compared NAVA with standard ventilation also reported use of a lower PIP $(p=0.02)$ with NAVA [21].

We acknowledge several study limitations. As a pilot effort, our study tested protocol feasibility and was not powered to detect a difference in the primary outcome. In fact, at the time we conceived this study, the literature provided no guidance concerning extubation success rates for NI-NAVA. Our protocol did not specify NI-NAVA management beyond the study period nor did it fully define ventilator management of infants extubated to NIPPV. Hence, variability in NIPPV management and/or physician bias due to the unblinded nature of the randomized treatment could have affected the primary study outcome and variability in management of both modalities might have affected secondary outcomes. The study lacked a sophisticated automated continuous analysis of the pressures and instead we relied on the EMR for PIP values averaged over each 24-h period.

In conclusion, the results of our pilot trial provide a rationale to conduct a larger adequately powered study to test whether use of NI-NAVA rather than NIPPV or nCPAP after initial extubation leads to a higher likelihood of extubation success and potentially to improved longer-term respiratory and other secondary outcomes. Our study demonstrated that a comparison trial was safe and feasible within our institution and supports future efforts to design and conduct the multicenter trial needed to confirm or negate our findings.
Acknowledgements We thank the families for trusting us with the care of their infants enrolled in this trial. We thank the three DSMC members Dr. Jennifer Liedel, Dr. Susan Aucott and Dr. Lily Lou for their inputs during the project to ensure subject safety.

\section{Compliance with ethical standards}

Conflict of interest The authors declare that they have no conflict of interest.

Publisher's note Springer Nature remains neutral with regard to jurisdictional claims in published maps and institutional affiliations.

\section{References}

1. Chawla S, Natarajan G, Shankaran S, Carper B, Brion LP, Keszler $\mathrm{M}$, et al. Markers of successful extubation in extremely preterm infants, and morbidity after failed extubation. J Pediatr. 2017;189:113-119.e2.

2. Bancalari E, Gerhardt T. Bronchopulmonary dysplasia. Pediatr Clin North Am. 1986;33:1-23.

3. Downing GJ, Hayen LK, Kilbride HW. Acquired subglottic cysts in the low-birth-weight infant. Characteristics, treatment, and outcome. Am J Dis Child. 1993;147:971-4.

4. Walsh MC, Morris BH, Wrage LA, Vohr BR, Poole WK, Tyson $\mathrm{JE}$, et al. Extremely low birthweight neonates with protracted ventilation: mortality and 18-month neurodevelopmental outcomes. J Pediatr. 2005;146:798-804.

5. Lemyre B, Davis PG, De Paoli AG, Kirpalani H. Nasal intermittent positive pressure ventilation (NIPPV) versus nasal continuous positive airway pressure (NCPAP) for preterm neonates after extubation. Cochrane Database Syst Rev. 2017;2:CD003212.

6. Donn SM, Sinha SK. Can mechanical ventilation strategies reduce chronic lung disease? Semin Neonatol. 2003;8:441-8.

7. Beck J, Campoccia F, Allo J-C, Brander L, Brunet F, Slutsky AS, et al. Improved synchrony and respiratory unloading by neurally adjusted ventilatory assist (NAVA) in lung-injured rabbits. Pediatr Res. 2007;61:289-94.

8. Allo J-C, Beck JC, Brander L, Brunet F, Slutsky AS, Sinderby CA. Influence of neurally adjusted ventilatory assist and positive end-expiratory pressure on breathing pattern in rabbits with acute lung injury. Crit Care Med. 2006;34:2997-3004.

9. Sinderby C, Beck J, Spahija J, de Marchie M, Lacroix J, Navalesi $\mathrm{P}$, et al. Inspiratory muscle unloading by neurally adjusted ventilatory assist during maximal inspiratory efforts in healthy subjects. Chest. 2007;131:711-7.

10. Beck J, Reilly M, Grasselli G, Mirabella L, Slutsky AS, Dunn MS, et al. Patient-ventilator interaction during neurally adjusted ventilatory assist in low birth weight infants. Pediatr Res. 2009;65:663-8.

11. Rossor TE, Hunt KA, Shetty S, Greenough A. Neurally adjusted ventilatory assist compared to other forms of triggered ventilation for neonatal respiratory support. Cochrane Database Syst Rev. 2017;10:CD012251.

12. Walsh MC, Kliegman RM. Necrotizing enterocolitis: treatment based on staging criteria. Pediatr Clin North Am. 1986;33:179-201.

13. Walsh MC, Yao Q, Gettner P, Hale E, Collins M, Hensman A, et al. Impact of a physiologic definition on bronchopulmonary dysplasia rates. Pediatrics. 2004;114:1305-11.

14. Jobe AH, Bancalari E. Bronchopulmonary dysplasia. Am J Respir Crit Care Med. 2001;163:1723-9.

15. Papile LA, Burstein J, Burstein R, Koffler H. Incidence and evolution of subependymal and intraventricular hemorrhage: a 
study of infants with birth weights less than 1500 gm. J Pediatr. 1978;92:529-34.

16. An international classification of retinopathy of prematurity. The committee for the classification of retinopathy of prematurity. Arch Ophthalmol. 1984;102:1130-4.

17. Fanaroff AA, Stoll BJ, Wright LL, Carlo WA, Ehrenkranz RA, Stark AR, et al. Trends in neonatal morbidity and mortality for very low birthweight infants. Am J Obstet Gynecol. 2007;196:147.e1-8.

18. Stoll BJ, Hansen NI, Bell EF, Shankaran S, Laptook AR, Walsh $\mathrm{MC}$, et al. Neonatal outcomes of extremely preterm infants from the NICHD Neonatal Research Network. Pediatrics. 2010;126:443-56.

19. Stein H, Beck J, Dunn M. Non-invasive ventilation with neurally adjusted ventilatory assist in newborns. Semin Fetal Neonatal Med. 2016;21:154-61.

20. Firestone KS, Beck J, Stein H. Neurally adjusted ventilatory assist for noninvasive support in neonates. Clin Perinatol. 2016;43:707-24.

21. Kallio M, Koskela U, Peltoniemi O, Kontiokari T, Pokka T, Suo-Palosaari M, et al. Neurally adjusted ventilatory assist (NAVA) in preterm newborn infants with respiratory distress syndrome-a randomized controlled trial. Eur J Pediatr. 2016;175:1175-83.

22. Chawla S, Natarajan G, Gantz MG, Shankaran S, Carlo WA. Reply. J Pediatr. 2018;194:263-4. https://doi.org/10.1016/j.jpeds. 2017.11.017.

23. Giaccone A, Jensen E, Davis P, Schmidt B. Definitions of extubation success in very premature infants: a systematic review. Arch Dis Child Fetal Neonatal Ed. 2014;99:F124-7.
24. Shalish W, Kanbar L, Kovacs L, Chawla S, Keszler M, Rao S, et al. The impact of time interval between extubation and reintubation on death or bronchopulmonary dysplasia in extremely preterm infants. J Pediatr. 2019;205:70-76.e2.

25. Shalish W, Kanbar L, Keszler M, Chawla S, Kovacs L, Rao S, et al. Patterns of reintubation in extremely preterm infants: a longitudinal cohort study. Pediatr Res. 2018;83:969-75.

26. Kirpalani H, Millar D, Lemyre B, Yoder BA, Chiu A, Roberts RS, et al. A trial comparing noninvasive ventilation strategies in preterm infants. N. Engl J Med. 2013;369:611-20.

27. Manley BJ, Doyle LW, Owen LS, Davis PG. Extubating extremely preterm infants: predictors of success and outcomes following failure. J Pediatr. 2016;173:45-49.

28. Jensen EA, DeMauro SB, Kornhauser M, Aghai ZH, Greenspan JS, Dysart KC. Effects of multiple ventilation courses and duration of mechanical ventilation on respiratory outcomes in extremely low-birth-weight infants. JAMA Pediatr. 2015;169:1011-7.

29. Donn SM, Sinha SK. Minimising ventilator induced lung injury in preterm infants. Arch Dis Child Fetal Neonatal Ed. 2006;91: F226-30.

30. Stein $\mathrm{H}$, Alosh $\mathrm{H}$, Ethington $\mathrm{P}$, White DB. Prospective crossover comparison between NAVA and pressure control ventilation in premature neonates less than 1500 grams. J Perinatol. 2013;33:452-6.

31. Lee J, Kim H-S, Sohn JA, Lee JA, Choi CW, Kim E-K, et al. Randomized crossover study of neurally adjusted ventilatory assist in preterm infants. J Pediatr. 2012;161:808-13.

32. Berger J, Mehta P, Bucholz E, Dziura J, Bhandari V. Impact of early extubation and reintubation on the incidence of bronchopulmonary dysplasia in neonates. Am J Perinatol. 2014;31:1063-72. 